\title{
Formas atípicas de presentación en pacientes con divertículo de Meckel. Reporte de casos
}

\section{Case Reports of Atypical Presentation of Meckel's Diverticulum}

\author{
Edward Ocampo Anduaga, MD, ${ }^{1}$ Alexis Ormeño Julca, MD, ${ }^{2}$ Jorge Reynoso Tantalean, MD, ${ }^{2}$ Leonardo Espinoza Solano, MD, ${ }^{2}$
}

Sandra Castillo Miranda, MD, ${ }^{2}$ Brenda Arévalo Porro, MD. ${ }^{2}$

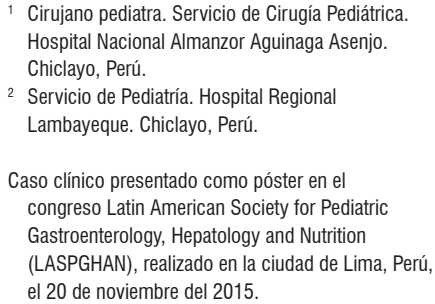

Caso clínico presentado como póster en el congreso Latin American Society for Pediatric Gastroenterology, Hepatology and Nutrition (LASPGHAN), realizado en la ciudad de Lima, Perú, el 20 de noviembre del 2015.

Fecha recibido: $\quad 05-04-16$ Fecha aceptado: 21-04-17

\section{Resumen}

El divertículo de Meckel es un vestigio embrionario del conducto onfalomesentérico, el cual normalmente regresiona en forma completa entre la quinta y la séptima semana de gestación. Se localiza en el borde antimesentérico del íleon y aproximadamente a $60 \mathrm{~cm}$ de la válvula ileocecal. Su incidencia es de $2 \%$ en la población general, con una relación varón-mujer de 2:1. Posee las mismas capas de la pared intestinal y en aproximadamente la mitad de los casos contiene tejido ectópico, que es gástrico en el 60\%-82\%, pancreático en $1 \%-16 \%$ y gástrico y pancreático en el $5 \%-12 \%$ de los casos. Tiene un curso asintomático y con mayor frecuencia es hallado durante laparotomías o necropsias. Aunque la gran mayoría de casos se manifiesta como hemorragia digestiva baja, existen formas menos frecuentes de presentación como obstrucción intestinal o perforación intestinal. La obstrucción intestinal asociada con el divertículo de Meckel puede ocurrir como resultado de la herniación o intususcepción alrededor del cordón fibroso que se extiende desde la pared abdominal al divertículo, del meso o del segmento intestinal, lo que puede llevar a una torsión obstructiva severa que provoca muchas veces necrosis y perforación.

Se presentan 5 casos de pacientes con diagnóstico de divertículo de Meckel con presentación atípica, 2 de ellos con hernia interna, 1 con invaginación intestinal y 2 con perforación bloqueada.

\section{Palabras clave}

Meckel, divertículo, obstrucción, intestinal, niños.

\section{Abstract}

Meckel's diverticulum is an embryonic vestibule of the vitelline duct which normally disappears completely between the fifth and seventh week of gestation. It is located at the anti-mesenteric ileal border approximately $60 \mathrm{~cm}$ from the ileocecal valve. Its incidence is $2 \%$ in the general population with a 2:1 male:female ratio. It has the same layers as the intestinal wall but contains ectopic tissue in approximately half of all cases: gastric tissue in $60 \%$ to $82 \%$, pancreatic tissue in $1 \%$ to $16 \%$ and gastric and pancreatic tissue in $5 \%$ to $12 \%$ of cases. It is often asymptomatic and is most frequently found during laparotomies and autopsies. While it is true that the vast majority of cases manifest as low gastrointestinal bleeding, there are less frequent forms of presentation such as intestinal obstruction and intestinal perforation. Intestinal obstruction associated with the Meckel's Diverticulum can occur as a result of herniation or intussusception around the fibrous cord that extends from the abdominal wall to the diverticulum, mesentery, or intestinal segment, which can lead to severe obstructive torsion that sometimes causes necrosis and perforation.

We present five cases of patients with Meckel's diverticulum with atypical presentations, two with internal hernias, one with intestinal invagination and two with blocked perforation.

\section{Keywords}

Meckel's diverticulum, obstruction, intestinal, children. 


\section{INTRODUCCIÓN}

El divertículo de Meckel es un vestigio embrionario del conducto onfalomesentérico. Frecuentemente se le hace referencia con la regla de los 2 (1):

- Presente en aproximadamente $2 \%$ de la población

- Relación varón/mujer 2:1

- Localizado a 2 pies $(60 \mathrm{~cm})$ de la válvula ileocecal

- Mide frecuentemente $2 \mathrm{~cm}$ de diámetro y 2 pulgadas (5 $\mathrm{cm}$ ) de longitud

- Puede contener 2 tipos de tejido ectópico (comúnmente gástrico y pancreático)

- Frecuente antes de los 2 años de edad

Cursa usualmente asintomático durante toda la vida, provocando síntomas solamente en el $1 \%$ de pacientes. Aunque mayormente se manifiesta como hemorragia digestiva baja, existen formas menos frecuentes de presentación como intususcepción, obstrucción intestinal, diverticulitis y perforación intestinal.

Se presentan 5 casos de pacientes con divertículo de Meckel con presentación atípica, atendidos en 2 hospitales de la ciudad de Chiclayo, Perú, entre los meses de enero a abril del 2015.

\section{CASO CLÍNICO 1}

Varón de 1 año que ingresa a emergencia pediátrica por vómitos alimentarios de 24 horas de evolución. Los exámenes auxiliares al ingreso mostraron leucocitosis en el hemograma, coagulograma sin alteraciones y función renal conservada. La radiografía de abdomen reveló una radiopacidad en cuadrante inferior derecho, con escasos niveles hidroaéreos. Fue evaluado por el cirujano e ingresó a sala de operaciones con el diagnóstico de obstrucción intestinal. Los hallazgos fueron: divertículo de Meckel a $80 \mathrm{~cm}$ de la válvula ileocecal, necrosado en su totalidad y con una brida en su extremo distal que lo unía a la pared abdominal.

Se realizó sección de la brida y resección de $6 \mathrm{~cm}$ de íleon, que contenían al divertículo de Meckel con posterior anastomosis ileal término-terminal, cursando con buena evolución posquirúrgica.

\section{CASO CLÍNICO 2}

Mujer de 8 meses de edad con antecedente de desnutrición crónica reagudizada que ingresa a emergencia pediátrica por vómitos y distensión abdominal de 3 días de evolución. La radiografía de abdomen mostró dilatación de asas intestinales y niveles hidroaéreos. Evaluada por cirugía pediátrica, se programó para laparotomía exploratoria por obstrucción intestinal encontrándose divertículo de Meckel a
$65 \mathrm{~cm}$ de la válvula ileocecal con brida en el extremo distal que la unía al mesenterio, creando un anillo herniario en cuyo interior se encontraba el íleon distal. Se realizó una resección ileal del segmento que contenía el divertículo (Figura 1) y anastomosis intestinal en un solo plano. El estudio anatomopatológico reveló el divertículo de Meckel con mucosa gástrica ectópica. Presentó durante la evolución dehiscencia parcial de anastomosis, por lo que requirió una nueva intervención quirúrgica para hacer drenaje e ileostomía. 4 meses después, se realizó la restitución del tránsito intestinal con una evolución favorable.

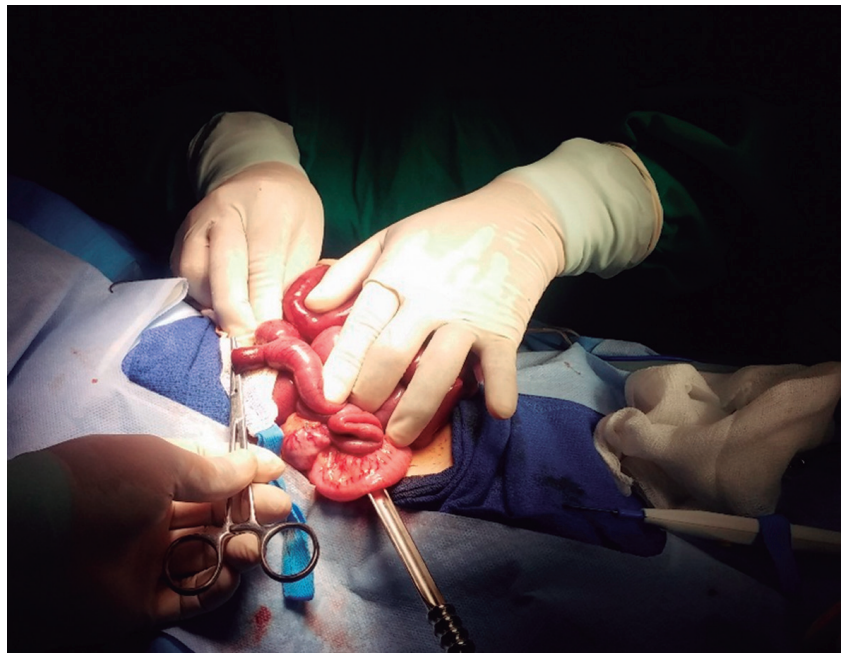

Figura 1. Divertículo de Meckel con brida en extremo distal que lo unía al mesenterio creando un anillo herniario en cuyo interior se encontró el íleon distal.

\section{CASO CLÍNICO 3}

Varón de 10 meses que ingresa a emergencia pediátrica con historia de vómitos y distensión abdominal de 2 días de evolución. La radiografía de abdomen mostró una dilatación intestinal y niveles hidroaéreos (Figura 2). Con el diagnóstico de obstrucción intestinal ingresa a la sala de operaciones donde se evidencia una invaginación intestinal, teniendo al divertículo de Meckel como cabeza de invaginación. Se procede a desinvaginación manual, resección de segmento intestinal que contiene al divertículo y anastomosis término-terminal. La evolución posoperatoria fue favorable.

\section{CASO CLINICO 4}

Varón de 1 año que ingresa a emergencia pediátrica por distensión y dolor abdominales, náuseas y vómitos de 36 horas de evolución.

La radiografía de abdomen mostró niveles hidroaéreos y ausencia de gas en la ampolla rectal. Ingresa a sala de ope- 


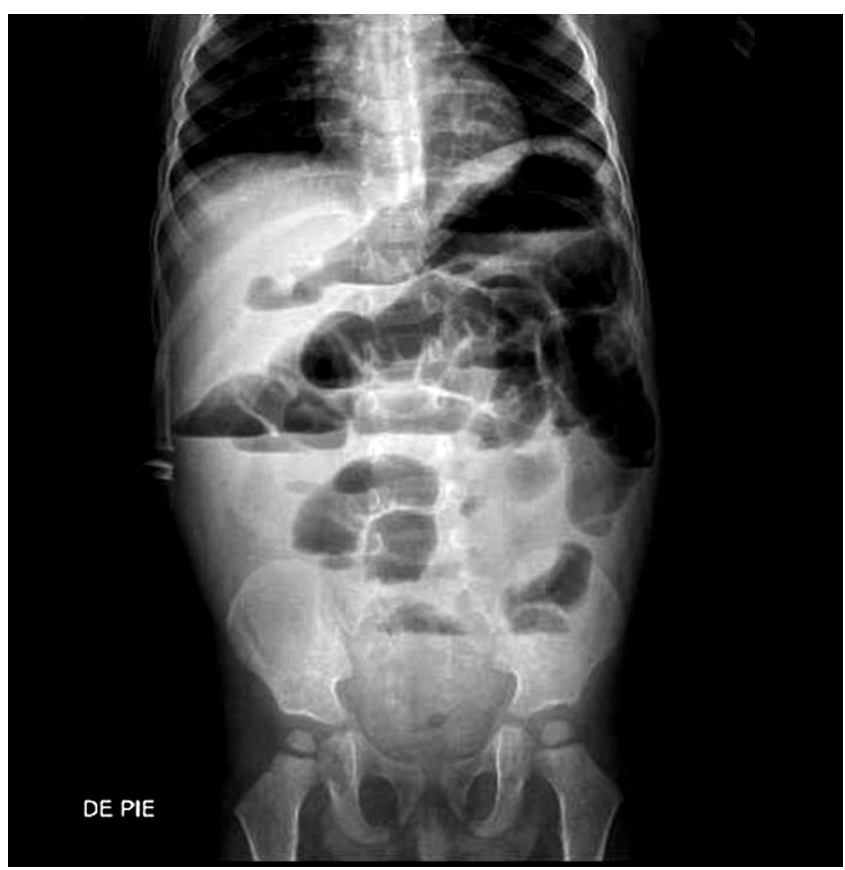

Figura 2. Radiografía anteroposterior (AP) de abdomen. Se observa dilatación de asas intestinales y algunos niveles hidroaéreos.

raciones con el diagnóstico de abdomen agudo y se halla un divertículo de Meckel a $60 \mathrm{~cm}$ de la válvula ileocecal con perforación bloqueada a ese nivel, lo que ocasionó una obstrucción intestinal. Se realizó una resección de divertículo de Meckel con anastomosis término-terminal con evolución posoperatoria favorable. El estudio anatomopatológico concluyó que era un divertículo de Meckel con mucosa gástrica ectópica (Figura 3).

\section{CASO CLÍNICO 5}

Mujer de 5 meses de edad, que ingresa a emergencia pediátrica con dolor y distensión abdominal progresiva de 5 días de evolución. 2 días previos al ingreso, se adiciona vómitos alimentarios y alza térmica no cuantificada. En el examen físico se encuentra el abdomen distendido, poco depresible y doloroso en forma difusa a la palpación. Ingresa a sala de operaciones con el diagnóstico de obstrucción intestinal. Los hallazgos fueron divertículo de Meckel perforado y con plastrón. Se realizó una resección y anastomosis con evolución favorable (Figura 4).

\section{DISCUSIÓN}

El divertículo de Meckel es la anormalidad congénita más frecuente del tracto gastrointestinal (TGI). Fue reportado por Guilhelmus Fabricius Hildanus en 1598, Levator en 1671, Ruysch en 1730 (2) y descrito en detalle por Johann Friedrich Meckel en 1808, por lo cual lleva su nombre.

Posee las mismas capas de la pared intestinal y en aproximadamente la mitad de los casos contiene tejido ectópico, siendo gástrico en el 60\%-82\%, pancreático en 1\%-16\% y de ambos tipos en el 5\%-12\% (3). Asimismo, se han encontrado neoplasias como tumores carcinoides, adenocarcinoma, tumor desmoplásico de células redondas y pequeñas $\mathrm{y}$ tumores mesenquimales benignos (lipoma, hemangioma y hamartoma) (4). Usualmente cursa asintomático y, por lo general, el diagnóstico se establece cuando se presentan complicaciones como hemorragia digestiva (20\%-30\%) (5), intususcepción, obstrucción intestinal por vólvulo o hernia interna, diverticulitis o perforación (6). La hemo-

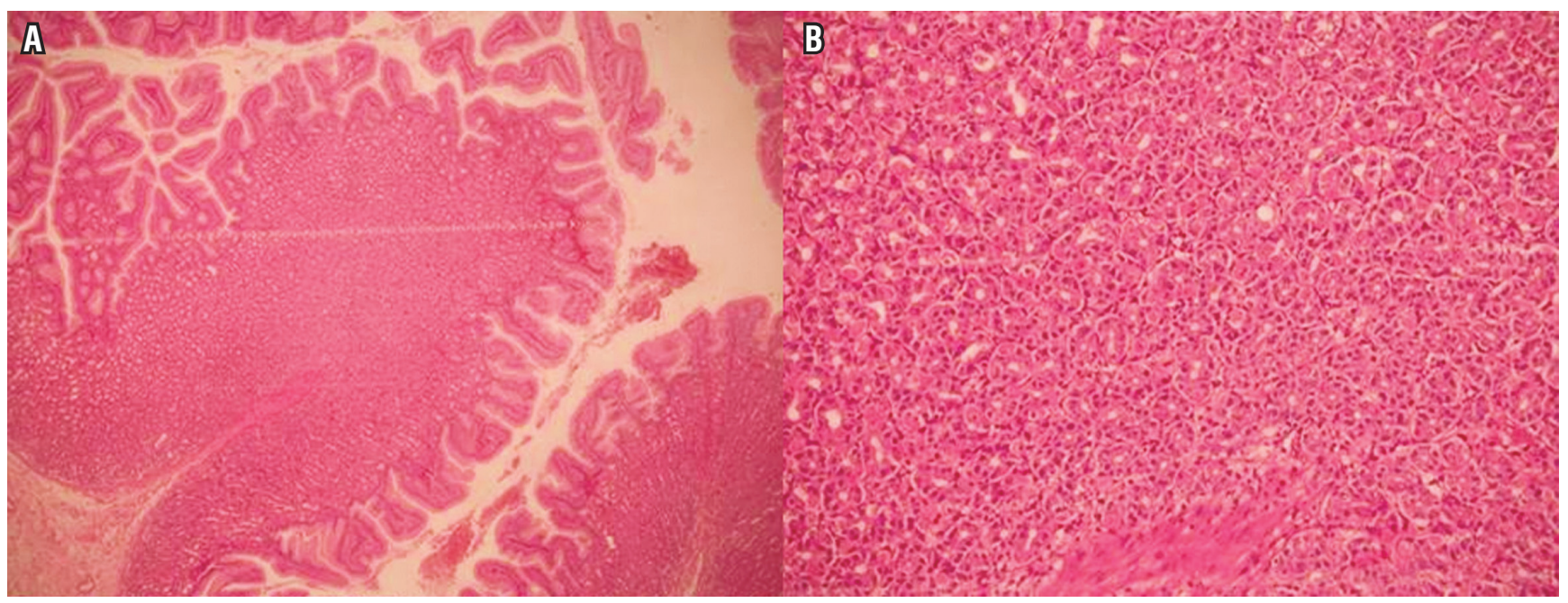

Figura 3. Histopatología del divertículo de Meckel. A: mucosa gástrica corporal ectópica. B: con mayor aumento se observan células parietales (tinción hematoxilina eosina). 


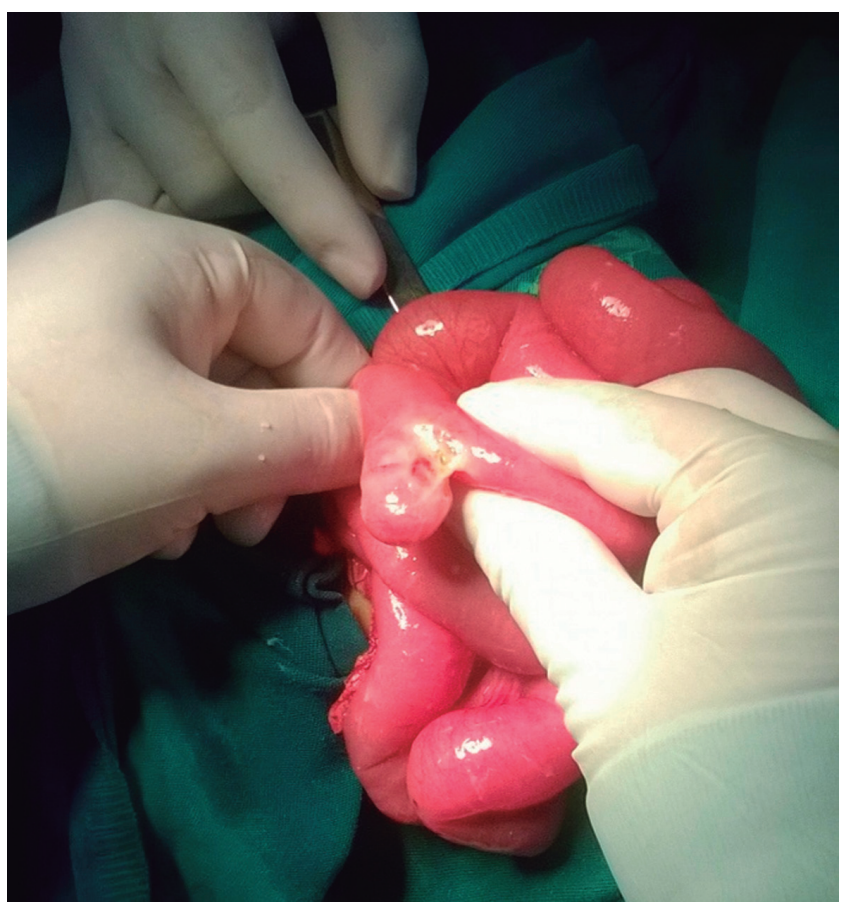

Figura 4. Divertículo de Meckel perforado formando un plastrón que provoca obstrucción intestinal.

rragia digestiva ocurre por ulceración péptica y ocurre frecuentemente en menores de 2 años (7).

La mortalidad y morbilidad reportadas en pacientes con divertículo de Meckel es del 6\%-7,5\% y del 6\%-30\%, respectivamente (3).

La obstrucción intestinal es un problema comúnmente encontrado en los servicios de emergencia pediátricos (8) y en el caso de divertículo de Meckel puede ocurrir como resultado de una intususcepción que tiene como cabeza de invaginación a este o por hernias internas, debido a que algunos divertículos presentan un cordón fibroso que los une al ombligo, lo que puede llevar a una torsión obstructiva severa provocando muchas veces necrosis y perforación, y también pueden deberse a bridas y adherencias secundarias a perforación. Aunque los casos 1 y 2 corresponden a pacientes con divertículo de Meckel complicado con una hernia interna, esta condición es sumamente infrecuente $(5,9,10)$ y usualmente compromete las regiones paraduodenal, foramen de Winslow, transmesentérica o transmesocólica. En caso de estrangulamiento de la hernia, el diagnóstico y tratamiento tempranos son esenciales debido a la alta tasa de mortalidad (aproximadamente 50\%) por descompensación hemodinámica $(11,12)$. La desnutrición causa afectación intestinal, con alteración de la absorción y la regeneración tisular, lo que aumenta el riesgo de complicaciones posquirúrgicas como dehiscencia de anastomosis, observado en el caso 2.
En el caso de invaginación intestinal, aunque la intususcepción idiopática es más frecuente entre los 3 meses y 3 años de edad, la intususcepción secundaria asociada con divertículo de Meckel ocurre en casi la mitad de los casos, en mayores de 3 años $(6,13)$. El caso 3 corresponde a un lactante con invaginación intestinal por divertículo de Meckel, que no solamente se encuentra en un grupo etario infrecuente para esta patología, sino que se presentó con clínica de obstrucción intestinal sin el hallazgo clásico de enterorragia. En diversos estudios, el hallazgo de divertículo de Meckel como desencadenante de la intususcepción no supera el 2,8\% de la totalidad de los casos en la edad pediátrica (14-16); sin embargo, estos datos no son aplicables a lactantes, pues las muestras no están centradas en este grupo y se sabe que en mayores de 2 años las alteraciones anatómicas son más frecuentes, lo que haría sobreestimar la incidencia.

La perforación o peritonitis por divertículo de Meckel, por lo general, se produce debido a una úlcera péptica originada por la secreción de ácido y pepsina por mucosa gástrica ectópica o secreción alcalina producida por tejido ectópico pancreático y el tratamiento consiste en una intervención quirúrgica inmediata con resección del divertículo o el segmento de íleon que lo aloja, tal como se realizó en los casos 4 y 5. Además, en pacientes con hemorragia se requiere resección intestinal segmentaria porque el sitio hemorrágico suele ser el íleon adyacente al divertículo (17).

El scan Meckel con tecnecio 99 es una herramienta diagnóstica ampliamente utilizada para identificar mucosa gástrica ectópica. Sin embargo, en pacientes con síntomas obstructivos, a pesar de su baja especificidad, se prefieren los estudios radiográficos o ultrasonográficos debido a que el divertículo de Meckel representa una causa infrecuente de obstrucción intestinal (6).

En los últimos años, la laparoscopia se ha constituido en una herramienta importante para el diagnóstico y el tratamiento del divertículo de Meckel en pediatría y se considera un procedimiento seguro para realizar la diverticulectomía, aun en casos complicados (18), prefiriéndose la diverticulectomía extracorpórea en comparación con la intracorpórea, debido a que la resección segmentaria del divertículo de Meckel puede ser realizado sin el uso de las costosas grapas laparoscópicas, además de que permite una completa extirpación no solo del divertículo, sino del tejido intestinal adyacente que contiene la mucosa ectópica para minimizar la posibilidad de recurrencia (19).

Asimismo, existen reportes del uso de la estereoscopia a doble balón con canalización ileal por colonoscopia inicial, como método diagnóstico para el divertículo de Meckel (20).

En conclusión, aunque en los casos de pacientes con divertículo de Meckel, la gran mayoría debuta con hemorragia digestiva baja, existen otras formas de presentación 
menos frecuentes que se deben tener en cuenta para incluir esta patología en el diagnóstico diferencial.

\section{REFERENCIAS}

1. Poley J, Thielen T, Pence J. Bleeding Meckel's diverticulum in a 4-month-old infant: treatment with laparoscopic diverticulectomy. A case report and review of the literature. Clin Exp Gastroenterol. 2009;2:37-40. Doi: https://doi. org/10.2147/CEG.S3792

2. Chaudhuri TK, Christie JH. False positive Meckel's diverticulum scan. Surgery. 1972;71(2):313.

3. Blevrakis E, Partalis N, Seremeti C. Meckel's diverticulum in paediatric practice on Crete (Greece): A 10-year review. Afr J Paediatr Surg. 2011;8(3):279-82. Doi: https://doi. org/10.4103/0189-6725.91665

4. Francis A, Kantarovich D, Khoshnam N, et al. Pediatric Meckel's Diverticulum: Report of 208 Cases and Review of the Literature. Fetal Pediatr Pathol. 2016;35(3):199-206. Doi: https://doi.org/10.3109/15513815.2016.1161684

5. Hawkins HB, Slavin Jr JD, Levin R, et al. Meckel's diverticulum. Internal hernia and adhesions without gastrointestinal bleeding - ultrasound and scintigraphic findings. Clin Nucl Med. 1996;21(12):938-40. Doi: https://doi. org/10.1097/00003072-199612000-00004

6. Huang C, Lai M, Hwang F, et al. diverse presentations in pediatric Meckel's diverticulum: a review of 100 cases. Pediatr Neonatol. 2014;55(5):369-75. Doi: https://doi. org/10.1016/j.pedneo.2013.12.005

7. Matsagas MI, Fatouros M, Koulouras B, et al. Incidence, complications and management of Meckel's diverticulum. Arch Surg. 1995;130(2):143-6. Doi: https://doi. org/10.1001/archsurg.1995.01430020033003

8. Blachar A, Federle MP, Dodson SF. Internal hernia: clinical and imaging findings in 17 patients with emphasis on CT criteria. Radiology. 2001;218(1):68-74. Doi: https:// doi.org/10.1148/radiology.218.1.r01ja5368 https://doi. org/10.1148/radiology.218.2.r01ja22384

9. Murakami R, Sugizaki K, Kobayashi Y, et al. Strangulation of small bowel due to Meckel diverticulum: CT findings. Clin Imaging. 1999;23(3):181-3. Doi: https://doi.org/10.1016/ S0899-7071(99)00113-8
10. Lee MC, Lin LH, Chen DF. Internal hernia caused by Meckel diverticulum in an infant: report of one case. Acta Paediatr Taiwan. 2001;42(2):105-7.

11. Martin LC, Merkle EM, Thompson WM. Review of internal hernias: radiographic and clinical findings. AJR Am J Roentgenol 2006;186(3):703-17. Doi: https://doi. org/10.2214/AJR.05.0644

12. Pershad J, Simmons GT, Chung D, et al. Two acute pediatric abdominal catastrophes from strangulated left paraduodenal hernias. Pediatr Emerg Care. 1998;14(5):347-9. Doi: https://doi.org/10.1097/00006565-199810000-00009

13. Fischer TK, Bihrmann K, Perch $M$, et al. Intussusception in early childhood: a cohort study of 1.7 million children. Pediatrics. 2004;114(3):782-5. Doi: https://doi. org/10.1542/peds.2004-0390

14. Kong FT, Liu WY, Tang YM, et al. Intussusception in infants younger than 3 months: a single center's experience. World J Pediatr, 2010;6(1):5-59. Doi: https://doi.org/10.1007/ s12519-010-0007-4

15. Jiménez J. Invaginación intestinal en pediatría. Rev Mex Cir Pediatr. 2005;12(4):195-203.

16. Shekherdimian S, Lee SL. Management of pediatric intussusception in general hospitals: diagnosis, treatment, and differences based on age. World J Pediatr. 2011;7(1):70-3. Doi: https://doi.org/10.1007/s12519-011-0249-9

17. Brañez C, Vargas B, Vargas J. Divertículo de Meckel perforado en un paciente de 13 años, reporte de un caso. Gac Med Bol. 2011;34(1):40-42.

18. Alemayehu H, Stringel G, Lo I, et al. Laparoscopy and complicated Meckel diverticulum in children. JSLS. 2014;18(3):e2014.00015.

19. Papparella A, Nino F, Noviello C, et al. Laparoscopic approach to Meckel's diverticulum. World J Gastroenterol. 2014;20(25):8173-8. Doi: https://doi.org/10.3748/wjg. v20.i25.8173

20. Qi S, Huang H, Wei D, et al. Diagnosis and minimally invasive surgical treatment of bleeding Meckel's diverticulum in children using double-balloon enteroscopy. J Pediatr Surg. 2015;50(9):1610-2. Doi: https://doi.org/10.1016/j.jpedsurg.2015.05.002 